\title{
DATA SLICING TECHNIQUE TO PRIVACY PRESERVING AND DATA PUBLISHING
}

\author{
Alphonsa Vedangi ${ }^{1}$, V.Anandam ${ }^{2}$ \\ ${ }^{I}$ Student, ${ }^{2}$ Professor, Department of CSE, CMR Institute of Technology, Hyderabad, Andhra Pradesh, India \\ alphonsa.vedangi@yahoo.com,velaanand@yahoo.com
}

\begin{abstract}
Many techniques have been designed for privacy preserving and micro data publishing, such as generalization and bucketization. Several works showed that generalization loses some amount of information especially for high dimensional data. So it's not efficient for high dimensional data. In case of Bucketization, it does not prevents membership disclosure and also does not applicable for data that do not have a clear separation between Quasi-identifying attributes and sensitive attributes. In this paper, we presenting an innovative technique called data slicing which partitions the data. An efficient algorithm is developed for computing sliced data that obeys l-diversity requirement. we also show how data slicing is better than generalization and bucketization. Data slicing preserves better utility than generalization and also does not requires clear separation between Quasi-identifying and sensitive attributes. Data slicing is also used to prevent attribute disclosure and develop an efficient algorithm for computing the sliced data that obeys ldiversity requirement. Experimental results confirm that data slicing preserves data utility than generalization and more effective than bucketization involving sensitive attributes. Experimental results demonstrate the effectiveness of this method.
\end{abstract}

Keywords -Privacy preserving, Data Security, Data Publishing, Microdata.

\section{INTRODUCTION}

Privacy preserving microdata publishing has been studied extensively in recent years. Today most of the organizations need to publish microdata. Microdata contain records each of which contains information about an individual entity, such as a person or a household. Many microdata anonymization techniques have been proposed and the most popular ones are generalization with k-anonymity and bucketization with 1diversity. In both methods attributes are into three categories, some of them are identifiers that can be uniquely identified such as Name or security number, some are quasi-identifiers. These quasi-identifiers are set of attributes are those that in combination can be linked with the external information to reidentify such as birth date, sex and zip code and the third category is sensitive attributes, this kind of attributes are unknown to the opponent and are considered sensitive such as disease and salary. These are three categories of attributes in microdata. In both the anonymization techniques first identifiers are removed from the data and then partitions the tuples into buckets. Generalization transforms the quasiidentifying values in each bucket into less specific and semantically constant so that tuples in the same bucket cannot be distinguished by their QI values. In bucketization, one separates the SA values from the QI values by randomly permuting the SA values in the bucket. The anonymized data consist of a set of buckets with permuted sensitive attribute values. The identity of patients must be protected when patient data is shared .Previously we used techniques using k- anonymity and 1-diversity. Existing works mainly considers datasets with a single sensitive attribute while patient data consists multiple sensitive attributes such as diagnosis and treatment. So both techniques are not so efficient for preserving patient data. So, we are presenting a new technique for preserving patient data and publishing by slicing the data both horizontally and vertically. Data slicing can also be used to prevent membership disclosure and is efficient for high dimensional data and preserves better data utility.

\subsection{Related Work}

To improve the disclosure of the patient data and to preserve better data utility sliced data is more efficient when compared to generalization and bucketization. In case of generalization $[29,31,30]$, it is shown that generalization loses considerable amount of information especially for high dimensional data. In order to perform data analysis or data mining tasks on the generalization table, the data analyst has to make the uniform distribution assumption that every value in a generalized set is equally possible and no other distribution assumption can be justified. This significally reduces the data utility of the generalized data. In generalizes table each attribute is generalized separately, correlations between different attributes are lost. This is an inherent problem of generalization. In case of bucketization, it has better data utility than generalization but does not prevent membership disclosure. Secondly bucketization publishes the QI values in their original forms, an opponent can easily find out whether 
an individual has a record in the published data or not. This means that membership information of most individuals can be inferred from the bucketization table. Also bucketization requires clear separation between QI and SI values .By separating the sensitive attributes from the quasi-identifying attributes, bucketization breaks the attribute correlation between the QIs and SAs. However in many data sets it is unclear that which attributes are QI's and which SA's are. So, bucketization also not so efficient for preserving microdata and publishing Slicing has some connections to marginal publication [15], both of them release correlations among a set of attributes. Slicing is quite different from marginal publication. First, marginal publication can be viewed as a special case of data slicing which does not have horizontal partitioning. Therefore correlations among attributes in different columns are lost in marginal publication.

\section{BASIC IDEAS OF DATA SLICING}

In this paper, we introduce a new method, called DATA SLICING. This method partitions the data both horizontally and vertically. Vertical partitioning is done by grouping attributes into columns based on the correlations among the attributes. Each column contains a subset of attributes that are highly correlated. Horizontal partitioning is done by grouping tuples into buckets. At last, within each bucket, values in each column are randomly permutated to break the association between different columns. The core idea of data slicing is to break the association cross columns, but to preserve the association within each column. This reduces the dimensionality of the data and preserves better data utility than bucketization and generalization. Data analysis methods such as query answering can be easily viewed on sliced data.

\subsection{Overview}

The overall method of slicing has been discussed above. The original microdata consist of quasi identifying values and sensitive attributes. In figure 1 patient data in a hospital. The data consists of Age, Sex, Zip code, disease. Here the QI values are $\{$ age, sex, zip code $\}$ and the sensitive attribute is \{disease\}.A generalized table replaces values

\begin{tabular}{|l|l|l|l|}
\hline Age & Sex & Zip code & Disease \\
\hline 22 & M & 47906 & Cancer \\
\hline 22 & F & 47906 & Thyroid \\
\hline 33 & F & 47905 & Thyroid \\
\hline 52 & F & 47905 & Diabetes \\
\hline 54 & M & 47902 & Thyroid \\
\hline 60 & M & 47902 & Cancer \\
\hline 60 & F & 47904 & Cancer \\
\hline
\end{tabular}

Figure 1: Original microdata published
In generalization there are several recodings. The recoding that preserves the most information is "local recoding". In local recoding first tuples are grouped into buckets and then for each bucket, one replaces all values of one attribute with a generalized value, because same attribute value may be generalized differently when they appear in different buckets.

\begin{tabular}{|l|c|l|l|}
\hline Age & Sex & Zip code & Disease \\
\hline$[20-52]$ & $*$ & $4790 *$ & Cancer \\
{$[20-52]$} & $*$ & $4790 *$ & Thyroid \\
{$[20-52]$} & $*$ & $4790 *$ & Thyroid \\
{$[20-52]$} & $*$ & $4790 *$ & Diabetes \\
\hline$[54-64]$ & $*$ & $4790 *$ & Cancer \\
{$[54-64]$} & $*$ & $4790 *$ & Nausea \\
{$[54-64]$} & $*$ & $4790 *$ & Cancer \\
{$[54-64]$} & $*$ & $4790 *$ & Thyroid \\
& & & \\
\hline
\end{tabular}

Figure 2: Generalized data

In bucketization also attributes are partitioned into columns, one column contains QI values and the other column contains SA values. In bucketization, one separates the QI and SA values by randomly permuting the SA values in each bucket. In some cases we cannot determine the difference between them two. So it has one drawback for microdata publishing. It also does not prevent membership disclosure

\begin{tabular}{|c|l|l|l|}
\hline Age & Sex & Zip code & Disease \\
\hline 22 & M & 47906 & Thyroid \\
22 & F & 47906 & Cancer \\
33 & F & 47905 & iabetes \\
52 & F & 47905 & Thyroid \\
\hline 54 & M & 47902 & Nausea \\
60 & M & 47902 & Thyroid \\
60 & M & 47902 & Cancer \\
64 & F & 47902 & Cancer \\
\hline
\end{tabular}

Figure 3: Bucketized data

Slicing does not require the separation of those two attributes. The basic idea of slicing is to break the association cross columns, but to preserve the association within each column. This reduces the dimensionality of data and preserves better utility. Slicing partitions the dataset both horizontally and vertically. Data slicing can also handle high-dimensional data. It provides attribute disclosure protection. 


\begin{tabular}{|c|l|}
\hline (Age,Sex $)$ & (Zipcode, disease) \\
\hline$(22, \mathrm{M})$ & $(47905$, Thyroid) \\
$(22, \mathrm{~F})$ & $(47906$, Cancer $)$ \\
$(33, \mathrm{~F})$ & $(47905$, Diabetes $)$ \\
$(52, \mathrm{~F})$ & $(47906$, Thyroid) \\
\hline$(54, \mathrm{M})$ & $(47904$, Nausea $)$ \\
$(60, \mathrm{M})$ & $(47902$, Thyroid) \\
$(60, \mathrm{M})$ & $(47902$, Cancer $)$ \\
$(64, \mathrm{~F})$ & $(47904$, Cancer $)$ \\
\hline
\end{tabular}

Figure 4: Sliced data

\section{SLICING ALGORITHM}

Step 1: In the initial stage we consider a queue of buckets $\mathrm{Q}$ and a set of sliced buckets SB. Initially Q contains only one bucket which includes all tuples and SB is empty. So $\mathrm{Q}=\{\mathrm{T}\} ; \mathrm{SB}=\varnothing$.

Step 2: In each Iteration the algorithm removes a bucket from $\mathrm{Q}$ and splits the bucket into two buckets. $\mathrm{Q}=\mathrm{Q}-\{\mathrm{B}\}$;

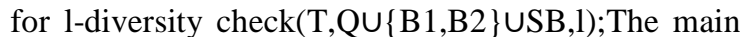
part of tuple partitioning algorithm is to check whether a sliced table satisfies 1- diversity.

Step 3: In the diversity check algorithm for each tuple $\mathrm{t}$, it maintains a list of statistics $\mathrm{L}[\mathrm{t}]$ contains Statistics about one matching bucket $B$. $t \in T, L[t]=\varnothing$. The matching probability $\mathrm{p}(\mathrm{t}, \mathrm{B})$ and the distribution of candidate sensitive values $\mathrm{D}(\mathrm{t}, \mathrm{B})$.

Step 4: $\mathrm{Q}=\mathrm{QU}\left\{\mathrm{B}_{1}, \mathrm{~B}_{2}\right\}$ here two buckets are moved to the end of the $\mathrm{Q}$

Step 5: else $\mathrm{SB}=\mathrm{SB} \cup\{\mathrm{B}\}$ in this step we cannot split the bucket more so the bucket is sent to SB

Step 6: Thus a final result return SB,here when $\mathrm{Q}$ becomes empty we have Computed the sliced table. the set of sliced buckets is SB .So, finally Return SB

\subsection{Algorithm for Diversity-Check}

Step 1: For each tuple $\mathrm{t} \in \mathrm{T}, \mathrm{L}[\mathrm{t}]=\varnothing$.

Step 2: For each bucket B in T.

Step 3: Record $\mathrm{f}(\mathrm{v})$ for each column value $\mathrm{v}$ in bucket B.

Step 4: For each tuple $t \in T$.

Step 5: Calculate $\mathrm{P}(\mathrm{t}, \mathrm{B})$ and find $\mathrm{D}(\mathrm{t}, \mathrm{B})$.

Step 6: $L[t]=L[t] \cup\{h p(t, B), D(t, B) i\}$.

Step 7: for each tuple $t \in T$.

Step 8: Calculate $\mathrm{p}(\mathrm{t}, \mathrm{s})$ for each $\mathrm{s}$ based on $\mathrm{L}[\mathrm{t}]$.

Step 9: if $\mathrm{p}(\mathrm{t}, \mathrm{s}) \geq 1 / \mathrm{L}$, return false.

Step 10: Return true,

\section{ATTRIBUTE DISCLOSURE PROTECTION}

Data slicing is used to prevent attribute disclosure, introducing the notion of 1-diverse slicing. The sliced table in figure 4 satisfies 2-diversity.considering tuple t1 with QI values(22,M,47906).In order to determine $t 1$ 's sensitive value one has to check t1's matching buckets. Consider an adversary who knows all QI values of $t$ and attempts to find out $t$ sensitive value from the sliced table. First let $p(t, B)$ be the probability that $\mathrm{t}$ is in bucket $\mathrm{B}$. Then the adversary computes $\mathrm{p}(\mathrm{t}, \mathrm{s})$, the probability that $\mathrm{t}$ takes a sensitive value $\mathrm{s}$. specifically let $\mathrm{p}(\mathrm{s} / \mathrm{t}, \mathrm{B})$ be the probability that $\mathrm{t}$ takes sensitive value $s$ given that $t$ is in bucket $B$,then according to law of total probability $\mathrm{p}(\mathrm{t}, \mathrm{s})$ is

$$
\mathrm{P}(\mathrm{t}, \mathrm{s})=\sum_{\mathrm{B}} \mathrm{p}(\mathrm{t}, \mathrm{B}) \mathrm{p}\left(\frac{\mathrm{s}}{\mathrm{t}}, \mathrm{B}\right)
$$

$$
P(t, B)=f(t, B) / f(t)
$$

Once we computed $\mathrm{p}(\mathrm{t}, \mathrm{B})$ and $\mathrm{p}(\mathrm{s} / \mathrm{t}, \mathrm{B})$ we can find out the probability $\mathrm{p}(\mathrm{t}, \mathrm{s})$ based on eq(1).

$$
\sum_{\mathrm{s}} \mathrm{p}(\mathrm{t}, \mathrm{s})=\sum_{\mathrm{s}} \sum_{\mathrm{B}} \mathrm{p}(\mathrm{t}, \mathrm{B}) \mathrm{p}\left(\frac{\mathrm{s}}{\mathrm{t}}, \mathrm{B}\right)=1
$$

SO 1-diverse slicing is based on the probability $\mathrm{p}(\mathrm{t}, \mathrm{s})$.A tuple $\mathrm{t}$ satisfies 1-diversity iff for any sensitive value $s, p(t, s)<=1 / L$.A sliced table satisfies 1-diversity iff every tuple in it satisfies 1diversity.our analysis directly shows that from an 1-diverse slice table, An adversary cannot learn the sensitive value of any individual with a probability greater than $1 / \mathrm{L}$.

\section{EXISTING SYSTEM}

We have many privacy preserving techniques and conducted extensive workload experiments. First, many existing clustering algorithms like k-means requires the calculation of centroids But there is no notion of centroids in our setting where each attribute forms a data points in the clustering space. Secondly, k-medoid method is very robust to the existence of outliers that is the data points that are very far away from the rest of the data points. Third, the order in which the data points are examined and does not affect the clusters computed from the k-medoid method.

\subsection{Disadvantages:}

1. Existing anonymization algorithms can be used for column generalization.

2. Existing data analysis such as query answering methods can be easily used on the sliced data.

3. Existing privacy measures for membership disclosure protection include differential privacy and presence.

\section{PROPOSED SYSTEM}

We present a novel technique called slicing, which partitions the data both horizontally and vertically. We show that slicing preserves better data utility than can be used for membership disclosure protection. Another important advantage of slicing is that it can handle high dimensional data. We show how slicing can be used for attribute disclosure protection and 
develop an efficient algorithm for computing the sliced data that obey the 1-diversity requirement. Slicing preserves better utility than generalization and is more effective than bucketization in workloads involving the sensitive attribute.

\subsection{Advantages:}

1. Slicing can be effectively used for preventing attribute disclosure based on the privacy requirement of 1-diversity.

2. Developing an efficient algorithm for computing the sliced table that satisfies the l-diversity. Proposed algorithm partitions attributes into columns applies column generalization and partitions tuples into buckets. Attributes that are highly correlated are in the same column.

\section{EXPERIMENTAL RESULTS}

Here we see how doctors and patients registers using their details based on quasi-identifying attributes and sensitive attributes. After registering doctor logins and see the patient published details based on their diseases.

\begin{tabular}{|c|c|c|c|c|c|}
\hline HOME & $\begin{array}{l}\text { DOTOR } \\
\text { REGISTER }\end{array}$ & $\begin{array}{l}\text { PATIENT } \\
\text { REGISTER }\end{array}$ & $\begin{array}{l}\text { DOCTOR } \\
\text { LOGIN }\end{array}$ & $\begin{array}{l}\text { PATIENT } \\
\text { LOGIN }\end{array}$ & $\begin{array}{l}\text { ADMIN } \\
\text { LOGIN }\end{array}$ \\
\hline \multirow{2}{*}{\multicolumn{6}{|c|}{$\begin{array}{l}\text { doctor register here } \\
\text { : }\end{array}$}} \\
\hline Doctor Id & & & & & \\
\hline Username & : & \multicolumn{2}{|l|}{ Shiva } & & \\
\hline Password & : & \multicolumn{2}{|l|}{ Shiva } & & \\
\hline Designation & : & \multicolumn{2}{|l|}{ MD } & & \\
\hline Gender & $:$ & \multicolumn{2}{|l|}{ M } & & \\
\hline Date of birth & : & \multicolumn{2}{|l|}{$21-7-1970$} & & \\
\hline Mobile & $:$ & \multicolumn{2}{|l|}{6756898467} & & \\
\hline City & : & \multicolumn{2}{|l|}{ Hyderabad } & & \\
\hline \multirow[t]{2}{*}{ Zipcode } & $:$ & \multicolumn{2}{|l|}{345678} & & \\
\hline & \multicolumn{2}{|c|}{ SUBMIT } & & & \\
\hline
\end{tabular}

Figure 7(a): Doctor Registration form 


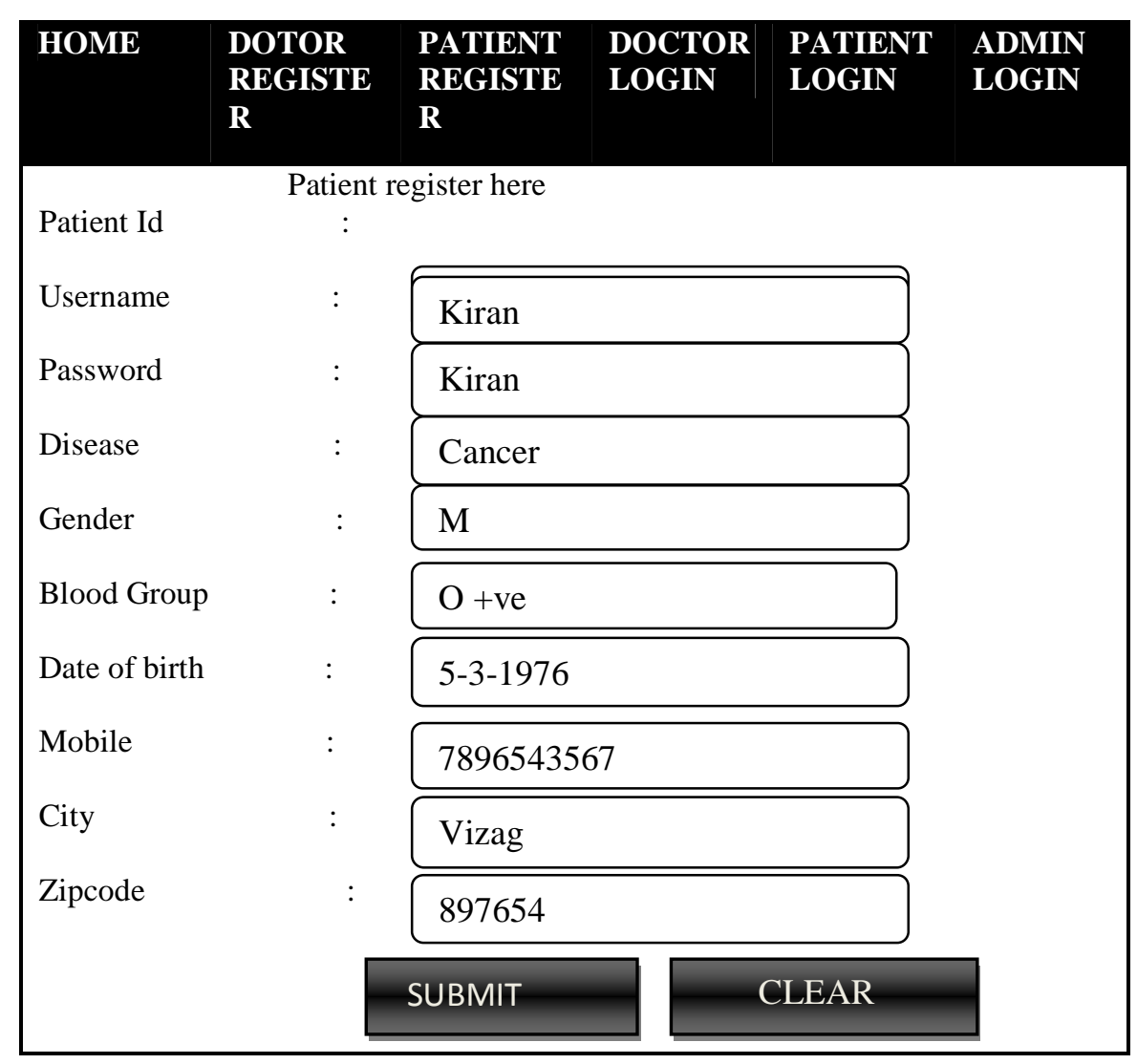

Figure 7(b): Patient registration forn

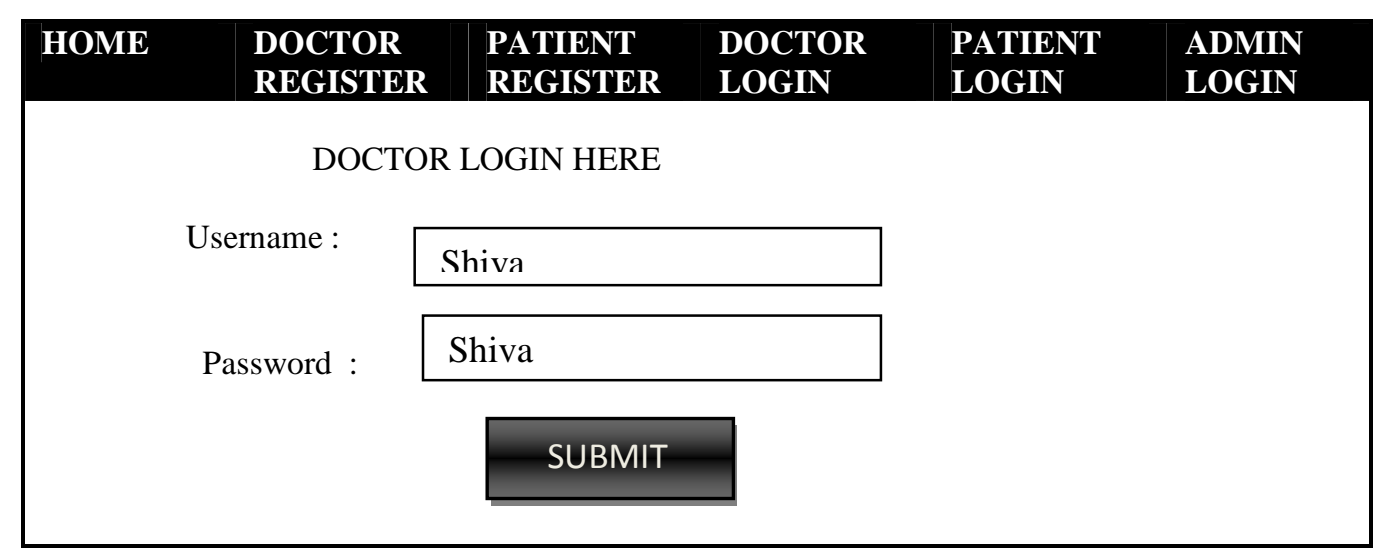

Figure 7(C): doctor login page

\begin{tabular}{|lllllll|} 
& HOME & $\begin{array}{l}\text { SEARCH } \\
\text { DISEASE }\end{array}$ & $\begin{array}{l}\text { COMMON } \\
\text { DATA }\end{array}$ & \multicolumn{2}{l|}{$\begin{array}{l}\text { CHANGE } \\
\text { PASSWORD }\end{array}$} & LOGOUT \\
\hline PATIENT ID & NAME & DISEASE & DOB & AGE & SEX & ZIPCODE \\
454 & Sita & Fever & $21 / 1 / 19^{* *}$ & $20-30$ & $\mathrm{~F}$ & $600^{* * *}$ \\
459 & Ali & Nausea & $11 / 7 / 17 * *$ & $40-50$ & $\mathrm{M}$ & $600 * * *$ \\
324 & Raju & Flu & $02 / 5 / 17^{* *}$ & $1-30$ & $\mathrm{M}$ & $479 * * *$ \\
\hline
\end{tabular}




\begin{tabular}{|c|c|c|c|c|c|c|c|}
\hline 765 & John & Cancer & $21 / 2 / 19 * *$ & $1-40$ & M & & $467 * * *$ \\
\hline 321 & Swathi & Fever & $14 / 5 / 19 * *$ & $10-30$ & $\mathrm{~F}$ & & $600 * * *$ \\
\hline 876 & Harika & Thyroid & $12 / 8 / 18^{* *}$ & $20-60$ & & $\mathrm{~F}$ & $600 * * *$ \\
\hline 564 & Amir & Cancer & $2 \quad 22 / 4 / 19 * *$ & $40-60$ & M & & $479 * * *$ \\
\hline 234 & David & Diabetes & $19 / 1 / 19 * *$ & $30-60$ & M & & $600 * * *$ \\
\hline 123 & Geeta & Nausea & $16 / 5 / 19 * *$ & $10-50$ & $\mathrm{~F}$ & & $600 * * *$ \\
\hline 932 & Latha & Thyroid & $22 / 8 / 19 * *$ & $120-70$ & & $\mathrm{~F}$ & $479 * * *$ \\
\hline 645 & Priya & Fever & $1 \quad 16 / 1 / 19 * *$ & $10-30$ & & $\mathrm{~F}$ & $479 * * *$ \\
\hline
\end{tabular}

Figure 7(d): Preserved data

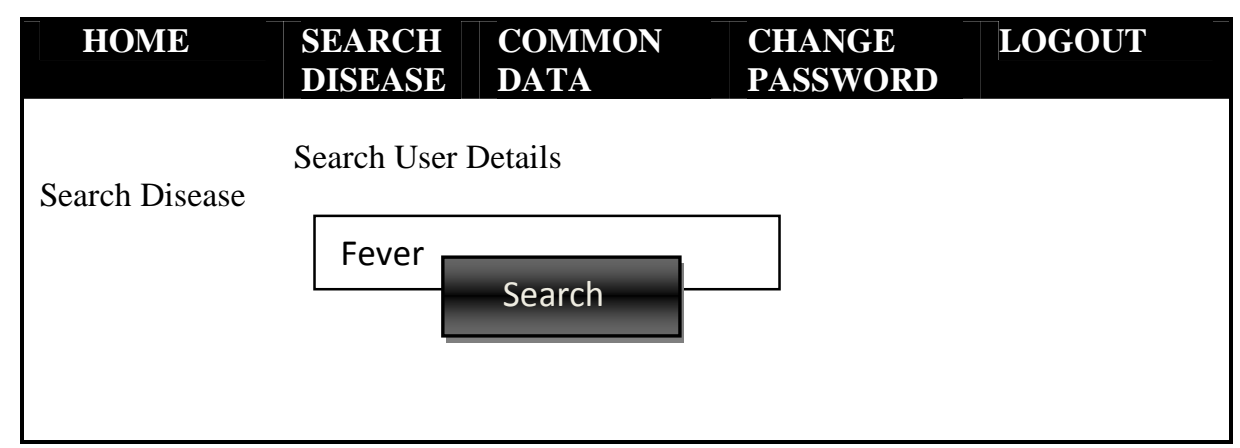

Figure 7(e): Data Searching

\begin{tabular}{|l|ll|llll|}
\hline & HOME & $\begin{array}{l}\text { SEARCH } \\
\text { DISEASE }\end{array}$ & $\begin{array}{l}\text { COMMON } \\
\text { DATA }\end{array}$ & \multicolumn{2}{l|}{$\begin{array}{l}\text { CHANGE } \\
\text { PASSWORD }\end{array}$} & LOGOUT \\
\hline & & & & & & \\
PATIENT ID & NAME & DISEASE & DOB & AGE & SEX & ZIPCODE \\
454 & Sita & Fever & $21 / 1 / 19 * *$ & $20-30$ & $\mathrm{~F}$ & $600^{* * *}$ \\
321 & Swathi & Fever & $14 / 5 / 19 * *$ & $10-30$ & $\mathrm{~F}$ & $600^{* * *}$ \\
645 & Priya & Fever & $116 / 1 / 19 * *$ & $110-30$ & $\mathrm{~F}$ & $479 * *$ \\
\hline
\end{tabular}

Figure 7(f): published data

\begin{tabular}{|c|c|c|c|c|}
\hline & HOME & DATA SLICED & $\begin{array}{l}\text { CHANGE } \\
\text { PASSWORD }\end{array}$ & LOGOUT \\
\hline PATIENT ID & NAME & A AGE,SEX & ZIPCODE,DISEASE & DELETE \\
\hline 454 & Sita & $(20-30, F)$ & (600045,Fever) & Delete \\
\hline 459 & Ali & $(40-50, M)$ & (600045,Nausea) & Delete \\
\hline 324 & Raju & $(1-30, M)$ & $(479801, \mathrm{Flu})$ & Delete \\
\hline 765 & John & $(1-40, M)$ & (467803,Cancer) & Delete \\
\hline 321 & Swathi & $(10-30, F)$ & (600045,Fever) & Delete \\
\hline 876 & Harika & $(20-60, \mathrm{~F})$ & & Delete \\
\hline 564 & Amar & $(40-60, M)$ & $\begin{array}{l}\text { (600032,Thyroid) } \\
\text { (479863,Cancer) }\end{array}$ & Delete \\
\hline 234 & David & $(30-60, M)$ & (600045,Diabetes) & Delete \\
\hline 123 & Geeta & $(10-50, F)$ & (600045,Nausea) & Delete \\
\hline 932 & Latha & $2 \quad(20-70, F)$ & $\begin{array}{l}1 \\
\text { (479650,Thyroid) }\end{array}$ & Delete \\
\hline 645 & Priya & $(10-30, F)$ & $(479650$, Fever $)$ & Delete \\
\hline
\end{tabular}

Figure 7(g): Sliced data 


\section{FUTURE WORK}

This proposed work motivates for several researches. Basically in this paper we considered slicing where each attribute is in exactly one column. The extension is the notion of overlapping slicing which releases more attribute correlations. One could choose to include the Disease attribute in the first column also and the privacy implications need to be carefully understood. This could provide better data utility. The tradeoff between utility of data and data privacy is very interesting. Finally, even though we are having many number of anonymization technique, it remains a problem how to use the anonymized data. In our work we randomly generated the associations between column values of a bucket.

\section{CONCLUSIONS}

In this paper, we present a new anonymization method that is data slicing for privacy preserving and data publishing. Data Slicing overcomes the limitations of generalization and bucketization and preserves better utility while protecting against privacy threats. We illustrate that how slicing is used to prevent attribute disclosures. The general methodology of this work is before data anonymization one can analyze the data characteristics in data anonymization. The basic idea is one can easily design better anonymization techniques when we know the data perfectly. Finally, we have showed some advantages of data slicing comparing with generalization and bucketization. Data slicing is a promising technique for handling high dimensional data. By partitioning attributes into columns, privacy is protected.

\section{REFERENCES}

[1]. G.Ghinita,Y.Tao, and P.Kalnis.On the anonymization of sparse high-dimensional data. In ICDE,pages 715$724,2008$.

[2]. A.Inan,M.Kantarcioglu,and E.Bertino.Using anonymized data for classification.In ICDE,2009 .

[3]. J.Brickell and V,Shmatikov.The cost of privacy:destruction of data-mining utlity in anonymized data publishing.In KDD,pages 70-78,2008.

[4]. J.Li,Y.Tao and X.Xiao.privation of proximity privacy in publishing numerical sensitive data. In SIGMOD,pages 473-486,2008.

[5]. R.C.-W.Wong,J.Li, A.W.-C. Fu, and K.Wang.( $\alpha, k)-$ anonymity: an enhanced k-anonymity model for privacy preserving data publishing.In KDD, pages 754$759,2006$.

[6]. V.Rastogi,D.Suciu,and S.Hong.The boundary between privacy and utility in data publishing.In VLDB,pages 531-542,2007.

[7]. T.Li and N.Li. On the tradeoff between privacy and utility in data publishing.In KDD,pages 517-526,2009.

[8]. K.LeFevre,D.DeWitt, and R.Ramakrishnan. Mondrian mulyidimensional k-anonymity.In ICDE,page 25,2006.
[9]. T.Li and N.Li. On the trade off between privacy and utility in data publishing .In KDD,pages517-526,2009.

\section{BIOGRAPHIES}

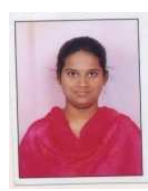

ALPHONSA VEDANGI, Department of Computer Science and Engineering, CMR Institute of Technology, Hyderabad With peer guidance from staff completed the research. Interested in researches and having first class aggregate till masters.

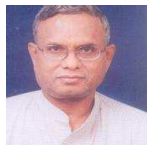

V.ANANDAM, B.E, M.S., Ph.D, Professor at CMR Institute of Technology, Department of Computer Science, Hyderabad Having Peer experience in teaching and administrative experience in academic colleges Coupled with 26 years of National and International project experience in high speed computer controls in process engineering 